\title{
Affording Illusions? \\ Natural Information and the Problem of Misperception
}

\author{
Hajo Greif \\ Warsaw University of Technology \\ International Center for Formal Ontology (ICFO) \\ Technical University of Munich \\ Munich Center for Technology in Society (MCTS) \\ mail@hajo-greif.net
}

Received 28 May 2019; accepted 20 September 2019; published 22 December 2019

\begin{abstract}
There are two related points at which James J. Gibson's ecological theory of visual perception remains remarkably underspecified. First, the notion of information for perception is not explicated in much detail beyond the claim that it "specifies" the environment for perception, and, thus being an objective affair, enables an organism to perceive action possibilities or "affordances." Second, misperceptions of affordances and perceptual illusions are not clearly distinguished from each other. Although the first claim seems to suggest that any perceptual illusion amounts to the misperception of affordances, there might be some relevant differences between various ways of getting things wrong. In this essay, Gibson's notion of "specifying" information shall be reconstructed with the help of Fred Dretske's relational theory of information. This refined notion of information for perception will then be used to carve out the distinction between perceptual illusions and the misperception of affordances, by reference to the "Empirical Strategy" in the psychology of perception (developed by Purves et al.). It will be maintained that there are cases where perceptual illusions actually help an organism to correctly perceive an affordance. In such cases, the prima facie mistaken or malformed informational relations involved are kept intact by a set of appropriate transformation rules. Two of Gibson's intuitions shall thus be preserved: the objectivity of informational relations and the empowerment of the organism as an active perceiver who uses those objective relations to his specific ends.
\end{abstract}

Keywords: ecological psychology; wholly empirical approach to perception; information for perception; affordance; perceptual illusions; psychology of perception 


\section{Introduction}

One of the most remarkable tenets of James Jerome Gibson's ecological theory of visual perception (1979) is the claim that information for perception "specifies" the environment for the organism and, in thus being an objective affair, enables direct perception of the "affordances" that some object or situation presents to him. In this paper, I will address two related questions raised by this assumption.

First, what notion of information is at work in the ecological theory? Gibson himself provides the reader with a mere few hints, yet given his view of information as being provided by "invariants" detectable in the "ambient energies," it bears two striking resemblances to Fred Dretske's otherwise quite differently motivated but more elaborate theory of information. It shares the Dretskean ontological virtue of treating information in relational terms instead of reifying it, and it spells out these relations as specification relations. However, it may also share some of the concomitant difficulties, most of all in accounting for the possibility of getting things wrong.

Hence, second, what status accrues to misperception and illusion? How can we get things wrong when our perception consists in picking up information that objectively specifies the environment and the qualities of the objects we perceive? Gibson acknowledges this problem but does not conclusively answer it. Moreover, he only discusses certain kinds of "illusion" and "misperception," and he uses these two terms in close association. However, there might be some relevant differences between the various ways of getting things wrong.

I will maintain that, under a well-reconstructed ecological perspective, misperception, as the misperception of affordances, should be kept clearly distinct from perceptual illusions. This distinction shall be carved out by reference to the "Empirical Strategy" (developed by Dale Purves and colleagues), a relatively recent approach in the psychology of perception, according to which there are adaptive functions to certain kinds of perceptual illusion. They may contribute to correctly guiding the perceiver's activities with respect to the object in question. Two of Gibson's intuitions shall thus be preserved: the objectivity of informational relations and the empowerment of the organism as an active perceiver who makes use of those relations.

Let me first briefly present my reading of the Gibsonian theory of perception and then address the two questions in proper order.

\section{Perceiving Affordances}

Perception, according to Gibson's view, does not amount to image-like representation of an outer physical world. One should not take experimental settings as the paradigm of perception, where the subject's visual apparatus is exposed to momentary stimuli detached from environmental settings ("snapshot" or "aperture vision," Gibson, 1979, p. 1). Nor do pictures or other mediated representations of world affairs provide a suitable paradigm of perception, as the information they provide is confined to a few aspects of their subject matter, allowing 
the viewer only to capture a limited subset of the information available in the environment (Gibson, 1979, Ch. 15). When moving in relation to a picture, one will discover the difference to a real scene with ease. It is the dynamics of spatial and somatic relations between perceiving organism and object that has to be systematically accounted for.

The idea that visual perception begins with the projection of an image onto the retina, Gibson holds, will be misguiding to begin with. It misguides us into believing, first, that we not only process such images but actually see them, or that some instance in our brain could see them, and make inferences - which Gibson derides as the "'little man in the brain' theory of the retinal image" (Gibson, 1979, p. 60). We might stop and reflect upon our perceptions by wondering what that red blot over here may be, or what shade of red it displays. However, part of Gibson's mission is to demonstrate that this is not how perception works. Second, and more subtly, the notion of the retinal image suggests that we perceive stimuli, and that perception is a response to those stimuli in which we derive information from them. Information would come into play, or even would be generated, only here, in the stages of perceptual processing. However, there are perfectly conceivable situations in which there is an abundance of stimuli that do not convey any information, such as in a brightly lit room filled with dense fog (Gibson, 1979, pp. 52-55; see the discussion in Chemero, 2003b) or, to use a more commonplace example, occurrences of so-called "whiteout" conditions, which can be dangerous to pilots, motorists or mountaineers precisely for the combination of a strong stimulus with the utter lack of visual information. Stimulus and information are in this sense detached, while stimulus and perception are not.

First and foremost, perception, on the Gibsonian view, is to be considered an activity that is intrinsically tied to other activities of an organism, and that depends on his general constitution and abilities on the one hand - his physiology, his body scale, the behaviours he is capable of and the resources he requires - and, on the other hand, on his current position and movements within his environment. Perception consists in the "pickup" of information from the "ambient energies" surrounding the organism. In relation to his position and movements, these energies form the "optic array" for perception (Gibson, 1979, Ch. 5). To accomplish the task of information pickup, visual and non-visual information about the position, orientation, and movement of the perceiving organism is included in the act of perception (Gibson, 1979, pp. 115-120).

In this fashion, information is actively retrieved from the environment, so as to detect patterns of persistence and change therein and track the "invariants" of some object. Invariants are to be understood in analogy to the mathematical meaning of the term (Gibson, 1971, p. 30; see also Gibson, 1973), as those properties of an object which remain unchanged when a set of rule-governed transformations is applied to it. For example, the length ratios of a geometric figure remain unchanged when it is scaled up and down proportionally. These ratios, but not the absolute measures of the figure's elements, are the object's invariants. In the context of perception, the transformations will encompass all naturally occurring changes in the conditions of perception, and the invariants will be what remains unchanged, as viewed in relation to the transformations of these conditions. In Gibson (1973, p. 45), 
we find a non-comprehensive list of candidate invariants, which comprises "alignment or straightness [...] as against bentness or curvature; perpendicularity or rectangularity; parallelity as against convergence; intersections; closures and symmetries." Citing Gibson's own example (1979, p. 13), a solid substance is rather persistent in shape, so shape is an invariant in the perception of all solid objects, but not in the perception of any less-thansolid object. For the latter, density or volume are likely to work as invariants.

The acts of retrieving information from the environment and hence tracking the invariants of some object or event do not involve the "replication" or "copying" of that object or event in the ambient light, as though some replica of the object were picked up in perception (Gibson, 1979, pp. 102-103). The tracking of invariants is not concerned with detecting similarities between an internal image and an object but with guiding the perceiving organism's activities towards that object. That guidance has to be accomplished throughout a multitude of transformations of conditions within the environment.

Above all, the organism's acts of perception imply the direct uptake of information on what can be done with a perceived object. Throughout all instances of being perceived, objects of all kinds, including other organisms, to use Gibson's most used (and often misused) concept, provide affordances to the perceiving organism. An affordance is what some object offers to be done with it, in the particular way in which it is related to the perceiving organism at a given time, under a given set of conditions. Accordingly, an object's affordance is always described in terms of possible actions of, or interactions with, the perceiving organism, such as standing, sitting, climbing up, jumping over, or falling off. For example, a fruit may afford activities such as being eaten, burrowing, being picked and used as a missile, or poisoning - or simply nothing at all. Affordances will vary between different animals for one and the same object, or even the same organism on different occasions. The activities an object affords to an organism constitute its specific "values" or "meanings," which can be directly perceived by the organism (Gibson, 1979, p. 127).

It would be unfortunate though to simply equate affordances with values and meanings. These are normatively highly charged terms that are likely to result in philosophical entanglements - which to avoid was one of the purposes behind the introduction of the concept of affordances (Gibson, 1966, p. 285). However, there are at least two related points at which that concept appears so vaguely circumscribed in Gibson's famous definition (1979, pp. 127, 129) as to invite such entanglements: There, he says that "affordances of the environment are what it offers the animal" (emphasis in original), but he also says that an affordance is "neither an objective property nor a subjective property; or it is both if you like" and that it is "equally a fact of the environment and a fact of behaviour." So, first, are affordances properties of objects, or are they better defined in terms of relations and situations? On an ecological view, objects and their properties might, and should, not be considered in detachment from the environments in which they are encountered, so Gibson's reference to objects and their properties should be read in light of this basic commitment. Second, are affordances entirely provided by the environment, or are they (partly) subjectively defined? If they are something that can be detected by an organism, 
they are supposed to be contained in the environment, but what is actually afforded in the act of perception can only be determined by including reference to the constitution and abilities of the perceiver. While the second apparent vagueness can be demonstrated to have a systematic purpose behind it, the first might be more difficult to parse.

Gibson's ambiguous characterization of affordances as properties and as facts might seem to be a minor issue, but the implications are significant if facts are taken to be comprised of things, properties and their relations: If affordances, their meanings and values were plainly and entirely properties of the environment and the objects therein, we would not only have to accept that such normative qualities were constituents of the environment; we would also end up in a world cluttered with an indefinite array of such qualities that would have to be embodied or embedded in the objects and the environments in which they are encountered, readily pre-packaged for all possible perceivers and detectable under all naturally occurring conditions of perception. These qualities might even contradict each other in certain cases, for certain perceivers, and still would have to be granted the same ontological status.

If one, disheartened by this prospect, does not want to settle for the opposite view that all normative qualities of some perceived object, and all variance therein, are located inside the organism proper and hence a subjective affair, an alternative is to locate values, their specificity to the perceiver, and their variance between perceivers in the relations between organism and environment. Affordances, as Gibson insists, are always related to activities, and are preferably described as capacities or abilities of the object with respect to the perceiving organism's constitution and abilities. The chair has the capacity of getting me seated, and the cliff has the ability to make me fall off. Even if we render these facts as seeming properties of fall-off-ability or sit-on-ability, these can only be defined in relation to the animal-with rather different outcomes for ants and primates. These relations will be best captured by a more complex, fact-like description, such as "the chair is sit-on-able for humans" or "the cliff is fall-off-able for large mammals".

Hence, it is a relational interpretation of affordances that appears best to make sense of that other, purposeful vagueness in Gibson's characterization of affordances as being objective and subjective at once. This relational, bi-directional interpretation can be further substantiated by an analysis of the nature of the information involved in affordances as being equally relational and bi-directional. This is what Gibson himself suggests when says that both affordances and information point "two ways, to the environment and to the observer" (1979, p. 141).

\section{Information}

On the reconstruction of Gibson's notion of perception as perception of affordances presented in the preceding section, it is both realist and anti-dualist in philosophical spirit. This reconstruction, I will continue to argue, should carry over to an account of ecological information for perception. There is one seeming ambiguity in Gibson's under-defined 
account of information that should be taken to be systematic. (There is no unequivocal definition of information in Gibson's 1966 and 1979 books, nor is there one in his smaller works on information, 1960 and 1971.) On the one hand, "information [is present] in ambient light to specify affordances" (Gibson, 1979, p. 143), and, as such, is present and specific even if and when an affordance is not perceived. On the other hand, information for perception is always comprised both of relations within the environment and of the perceiving organism's relations to his environment. Hence, an important part of information for perception is dependent on the perceiving organism, and all information for perception is relational in kind. Within ecological psychology, there has been a variety of diverging interpretations of the relation between Gibsonian affordances and informationsome of which may help to identify what is at issue here.

Edward Reed (1988) is peculiar in taking ecological information and affordances to be properly external to the perceiving organism, as doing otherwise, he argues, might invite the subjectivism expounded by theories of indirect perception. Moreover, Reed takes affordances to be "the functional properties of objects as, for example, the affordance of a heavy stick or rock for pounding" (1988, p. 231). Prima facie, this definition looks quite similar to the one provided by Harry Heft (2001, p. 124): "an affordance is a property of the environment that has perceived functional significance for an individual." (Note that, on a literal reading of this definition, an affordance only exists if and when it is actually perceived.) However, Heft also refers to affordances as relational properties of the environment, that is, properties that can only be determined in relation to the organism. In contrast to Reed, then, affordances and information are not deemed external to the organism while remaining defined in terms of properties.

In expressly anti-dualist spirit, Michael Turvey (1992) refers to affordances as dispositional properties of objects that are actualized under a concrete set of conditions. Turvey later refers to affordances as "single complex particulars," in which co-ordinations of situations, activities, organisms and concrete occasions are what constitutes perception and action (2013). This notion of complex co-ordinations contributes to de-emphasizing the organism/environment distinction and avoids standard physicalistic views of how the environment stands. It even reverses the explanatory burden that is normally presumed when matching perception against physical measurements: Why should our physics diverge from how we perceive things in the first place, and why does it remain ambiguous on many points? ${ }^{1}$ A reification of affordances as envisioned by Turvey requires, and is duly accomplished by, a rather thick ontology that is able to collapse complex facts, constituted by objects, their properties and relations, into singular properties with a certain hue of potentiality.

In contrast to these "property" views, Anthony Chemero (2003a) develops a relational theory of affordances that is complemented by an equally relational account of information for perception (Chemero, 2003b). Whereas relations exist between entities with certain properties, this does not entail that these relations are properties themselves. For example, John

\footnotetext{
${ }^{1}$ This point was raised by Michael Turvey (personal communication).
} 
is taller than Sally. Quite obviously, "being taller than" is neither an absolute measure, nor is there a complex property of taller-than-ness, let alone taller-than-Sally-ness possessed by John (Chemero, 2003a, p. 187). If we conceive of information as informational relations from the start, we do not incur the ontological challenge of allowing for such complex properties. If perception of an affordance is based on relations between the ambient energies and structures in the environment, including the perceiving organism himself (Chemero, 2003b, pp. 580-581), an affordance is, in Chemero's wording, a relational "feature" of entire "situations" of an organism moving in and through a certain environment.

Only partly buying into this relational account of information and affordances, Alan Costall (2004) argues that Gibson sought to define information without reference to a perceiver while insisting that affordances can only be defined by reference to the perceiver. He diagnoses a shift in views on perception between Gibson's earlier and later works. First, there is the claim that perception of affordances is based on information contained in the optic array. Second, there is the claim that affordances, and hence the specific meanings of an object to a perceiving organism, essentially depend on the organism's constitution and abilities, and can only be relationally defined. Costall sees an essential tension in Gibson between taking information to be objectively 'out there' (and, by implication, excluding the perceiver) and a functional concept of information according to which it can only be defined relationally, in view of an affordance to a specifically constituted organism-which he takes to be the tenable position.

In order to resolve that same ambiguity, Ludger van Dijk, Rob Withagen and Raoul Bongers (2015) argue that information for perception in ecological psychology is best understood as being devoid of semantic content. The information picked up by a perceiving organism is not about an affordance, and hence a semantic relation, which they fear would undermine the purpose of a theory of direct perception by smuggling in the baggage of mental representations. Instead, information should be understood functionally, as being for an affordance, where that function is realized in the use of the information involved, and hence in the perceiving organism's activities. At the same instance, van Dijk et al. (2015), like Chemero (2003a), Costall (2004), and Heft (2001, Ch. 3) before them, all acknowledge what Gibson (1979, p. 115-116) explicitly maintains (and what Reed [1988] seems to ignore): that the information picked up in perception does include relations between organism and environment. It specifies the environment and the self, and hence can be both an objective commodity and relationally defined.

A more detailed and systematic reconstruction of this dual, relational nature of information for perception can be derived from a theory of information that comes from a background and with intentions quite distinct from ecological psychology: In Knowledge and the Flow of Information, Fred Dretske (1981) develops a theory of information designed to support a naturalistic approach to philosophical semantics, in which he makes approving reference to Gibson's theory. Remarkably, and unlike much of philosophical semantics, Dretske's theory of information does not presuppose meaning but seeks to explain its emergence 
from informational relations. On Dretske's account, information is a certain relation between two world affairs $s$ and $r$ in which $r$ is a signal of the other affair $s$ having some specific property $F$. Dretske, unlike Gibson, does dare to talk about signals, transmission and receivers, but he does so in an expressly deflationary spirit. An informational relation is the case only if and only when the signalled affair of $s$ having that property $F$ is the case whenever the signalling affair $r$ occurs, so that the conditional probability for that relation to hold in any given situation is 1 . Moreover, $r$ must be tied to the affair at the information source by some, at root nomological, regularity. Coincidentally parallel transformations of values at $s$ and $r$ will not count, even if the coincidence is perfect and persistent. The regularity and unequivocality of these relations are the mark of Dretskean information. This is what qualifies it as a template for a more precise definition of Gibsonian information as a specification relation.

Dretske's view of information presents a vivid contrast to the paradigm of information embodied by the Shannon-Weaverian mathematical theory of communication and the doctrines contained therein, even though he borrowed his terminology from it. ${ }^{2}$ It does so in at least three respects. To begin with, Dretske's notion of information is concerned with identifying the informational content of some signal and hence its meaning, whereas the mathematical theory of communication mostly confined itself to the measurements of signal strength and channel conditions, as they are required for successful transmission. Second, Dretskean information definitionally does not presuppose the presence of a sender or receiver - which are basic units in the mathematical theory of communication. Of course, some of the correlations involved will be important to receivers and, in a subset of cases, senders, but information is supposed to be present in the environment without them. Third, Dretskean information is defined in purely relational terms. Information is a probabilistically described (but nomologically governed) relation between world affairs that does not add anything, ontologically, to a natural environment and that assumes its status as information long before and sometimes without ever becoming processable by some appropriate machinery, organic or other. To Dretske, the relations and regularities he describes are not only necessary but also sufficient for the presence of information. To the extent that reified entities, substances and properties, enter into informational relations, these are the $r$-tokens and the $F$-conditions at $s$, not the relations themselves.

Although he does not clearly define it as such (and although Fodor and Pylyshyn, [1981, pp. 166-167] claim otherwise), Gibson appears to employ a similarly relational, non-reified view of information when he discusses the perceiving organism's tracking of the invariants of some object in the environment and the activity of extracting information from the ambient optic array. If that information is supposed to remain identical throughout the

\footnotetext{
${ }^{2}$ Less charitably, it has been argued that Dretske's reference to the mathematical theory of information is tenuous or even purely rhetorical, and hence might misguide the reader, and that his liberal re-definitions of informationtheoretical terms do not help either (see, for example, Sayre, 1983, p. 79). Ruth Millikan (personal communication) holds a similar view.
} 
various instances and situations of perception, it has to be regular in a fashion that is independent of subjective conditions of perception - even though the perceiving organism's position, constitution and abilities will enter into the set of perceivable relations. As these relations remain stable and unequivocal in an otherwise changeable environment, he can relate to them in various ways over time, under variant conditions. While organism-related variables enter into a given set of informational relation that he uses, the functional status of his sensory organs or his degree of attentiveness in a given perceptual situation will not.

The invariant character of informational relations in the environment is foundational to the possibility of keeping track of objects and events throughout a multitude of conditions for a variety of differently constituted organisms, and hence for providing affordances that are organism-specific. An object affords what it affords because some of its properties remain stable and reliably detectable in the ambient optic array for the perceiving organism. They are reliably detectable precisely if there are, first, conditions for perception which are such that any transformation of the object or its position has strict and unequivocal correlates in its placement in the ambient optic array. Second, the perceiving organism is able to match his position, movements, and aims against the relevant environmental invariants over time, thereby using proprioceptual information. Both sets of relations in conjunction are the informational relations that specify, in Gibson's phrasing, the environment for the organism. Third, the organism must be in a condition suitable to actually picking up that information. Precisely if and when both the environment - and the organism-bound conditions are fulfilled, there will be a direct perception of affordances: The perceiving organism is enabled to immediately rely on the relation between himself and what remains invariant, what varies regularly and what varies arbitrarily in an object over the course of his perceptual activities.

By including organism-related variables, information in the ecological sense is "intrinsic" to a perceptual situation, as distinguished from "extrinsic" information that allows for absolute, perceiver-independent measurements of physical and physiological variables by an external observer. Intrinsic, affordance-related information still allows for measurement, although on a different basis (see Gibson, 1979, p. 128). The paradigm of measurementoriented approaches in post-Gibsonian ecological psychology are the measurements of stair-climbing affordances for persons of different leg lengths in William J. Warren's study (1984, where the intrinsic/extrinsic distinction is introduced; see also Boumans, 2013). Although there is considerable variance in the relation between riser heights and leg length for short and tall persons, the results of the experiments in this study suggest that there are ratios for stair-climbing affordances that remain constant across the study population. These ratios can be expressed in an intrinsic or body-scaled metric that matches the dimensions of the subjects' bodies and the dimensions of the object in question - in this case: the riser height of the stairs. Such ratios can be found both for perceptual category boundariesbetween stairs that are perceived as still being climbable and stairs that are already perceived as being unclimbable - and for optimal heights in terms of effort to be invested into climbing. The ratios are such that short people will perceive the same affordance for relatively low risers that will be perceived by tall people for proportionally higher risers. The 
ratios however do not express subjective factors, in that they remain constant across the population, with identical absolute measures for people of the same size, and in that perceived optimal heights quite closely match the actual energetic optima in physiologically based trials. Hence, Warren concludes, environmental objects are perceived by an organism in relation to his action capabilities, where that relation is rather stable and unequivocal.

On this background, affordances can be relationally defined, in a two-step fashion that builds upon informational relations and the perceiving organisms' abilities to track, and act upon, these relations - which will include their own specific relations to their environment. This relational view allows for a fairly clear set of distinctions to be made with respect to situations in which an affordance, as it is perceived by an organism, and information for perception do not appear to match: There is no information if the relations between signalling and signalled world affairs are not unequivocal, even if and when an organism appears to track such a relation, so there is no affordance to be perceived either. Conversely, if there is a malfunction in an organism's perceptual system, information will still be present in the environment, as there will be nomologically governed relations between incoming light, refraction and reflection on surfaces, etc., but these relations could not, or not properly, be detected by the perceiving organism for reasons internal to him. Hence, the third of the above requirements, namely that of being in a condition suitable to picking up information, will be violated. This condition will be violated in a different manner if modifications or untypical conditions in the environment intervene, to the effect that the relations involved are not within the range of what is detectable even for a properly functioning perceptual system despite being nomological in kind.

A markedly different route to accounting for mismatches between affordances and information has been taken by critics of Dretske's and Gibson's (partly implicit) accounts of information who argue that information, although being provided by the environment, is not a specification relation (see Millikan, 2001, with focus on Dretske, and de Wit, van der Kamp, \& Withagen, 2015; Withagen \& Chemero, 2009, with focus on Gibson). These critics suggest a 'softer' notion of natural information as the biologically more realistic one: "epistemic contact" with the environment, in Withagen and Chemero's wording, typically comes in degrees. Perfect contact, that is, full specification, is practically unattainable for organisms under natural conditions of perception, and hence will pose too high a requirement. However, de Wit et al. (2015) argue with reference to Millikan (2001), direct perception of affordances may not depend on full specification but allow for more probabilistic and local relations, as long as these are satisfactorily reliable for an organism.

Given that organisms are always bound to locally and temporally variable environmental conditions, this argument has some prima facie plausibility. As de Wit et al. (2015) admit, locating ambiguities in perception in the informational relations themselves will be a concession to "inferential" approaches to perception, for the sake of ecological credibility. However, achieving such credibility does not require one to accept the inferentialist "doctrine of intractable nonspecificity" of informational relations (as Turvey and Shaw [1979] call it). If informational relations may hold between all sorts of world affairs, and if they 
are regular in the way outlined by Dretske, there will be relations in the environment that remain fully specific even under changeable ecological conditions. Perceiving organisms, being finite creatures with finite resources acting within concrete ecological situations, are in practice not in a position to track all those specifying relations. Gibson himself admits that information may be inadequate, impoverished or masked in a given perceptual situation, so that conditions for perception fall out of the range of variance that an organism is accustomed to (Gibson, 1966, Ch. 14). Such will be the case when unusual lighting conditions obtain or when distorting mirrors or similar devices are placed in the environment. These qualifications, however, apply to what the perceiving organism is in a position to pick up from his environment rather than to the specificity of information as such.

In developing this account of "misrepresentation," Dretske (1986) seeks to accommodate for the possibility that an organism might get things wrong despite informational relations being in proper shape. Under normal conditions, he receives information on some world affair through several independent channels, and will be able to evaluate some signal or channel condition and remedy against what a faulty, information-less signal might seem to convey. Although he carefully avoids information-theoretical talk of "channels", and although he avoids referring to successful and unsuccessful perception in terms of truth and falsity of representations, Gibson apparently holds a similar view when he refers to information as being redundantly available in the environment, allowing for "multiple specification" (as paraphrased by Runeson [1988, p. 296-297]). Still, Gibson can be seen struggling with the concept of misperception (see Gibson, 1979, pp. 243-244). Unlike Dretske, he accepts that misinformation could still be information while he insists, like Dretske, that one will need separate theories of successful and unsuccessful perception respectively (Gibson, 1966, pp. 287-288). Hence, the parallel to Dretske's (1986) view that a theory of information cannot be symmetrically applied to cases of misinformation is only partial. Hence, we are now landing in the middle of the second problem identified in the introduction: How do we account for illusion and misperception if information is an objective commodity and perception is the activity of tracking the relations that make up that commodity?

\section{Perceptual Illusions}

On some accounts, the possibility of misperception and illusion appears to be a hard, and possibly insurmountable, problem for an ecological theory of perception (see, first and foremost, Fodor \& Pylyshyn, 1981, pp. 153-155). A short answer to this apparent problem is offered in a reply to Fodor and Pylyshyn from the camp of ecological psychology. It says that the Gibsonian conception of information "is roughly the claim that real possibilities are specified by current states of affairs." (Turvey, Shaw, Reed, \& Mace, 1981, p. 293) On this view (which is largely shared here), the requisite information is present only if the affordance or "possibility" in question is real, and hence is warranted by current states of affairs. Sometimes, the perceiving organism will have to find out the hard way whether a possibility is real, and whether the information he seemed to pick up is there at all. Quite often, however, he has means of reliably making that distinction in the course of perceptual activity. 
There is a variety of ways of being mistaken about some world affair, which are only partly acknowledged by Gibson. In his Ecological Approach, he briefly and tangentially refers to misperception and perceptual illusions, and he does so primarily in the context of artfully created illusions. His prime examples are of two kinds:

(pi 1) Pictures that are purposefully made to create the appearance of objects that are not present in the environment (Gibson, 1979, pp. 281-283).

Hence, it is quite remarkable to find pictures subsumed under illusions, but this claim is consistent with Gibson's theory, and its normative connotations are not negative by default. A picture of some concrete object will contain some of the information that would be necessary for the object in question to provide the requisite affordance to its perceiver in its proper environment, yet without actually providing that affordance-for example, the sitting-on and sitting-at detectable but unavailable for the picture of a chair and a table. In such cases, we normally recognize both that would-be affordance and the pictorial nature of what is presented to us, and are therefore not misguided. We are able to do so primarily because we actively explore what we perceive, by moving our eyes and heads in relation to an object. Only if and when we did not "test for the reality" of what we perceive, there could be such a thing as a perfect picture that tricked us into mistaking it for the scene that is being pictured (Gibson, 1970, p. 426). ${ }^{3}$

(pi 2) Devices that are purposefully placed in the environment so as to create discontinuities in perception.

Drawing on the experimental work of his wife, Eleanor J. Gibson (Gibson \& Walk, 1960), Gibson's paradigm of such artfully created illusions are planes of solid glass that extend over visual cliffs and thus provide support while maintaining the visually based affordance of falling off the cliff (Gibson, 1979, pp. 142-143). Here, conditions are modified in such a way as to create a mismatch between current states of affairs and an affordance or "possibility", which thus becomes "unreal". Misguiding the subject's perception is one central aim of the experiment, so the purpose of the artefact is clearly at variance with (pi 1).

These two cases, being quite distinct affairs themselves, are rather different, but not clearly distinguished by Gibson, from two other kinds of cases:

(pi 3) Naturally occurring perceptual illusions, such as illusions of length, colour or brightness.

(pi 4) Instances of misperceiving an object for another object that would have afforded different activities to the perceiver.

\footnotetext{
${ }^{3}$ Several years prior to the Ecological Approach, Gibson was involved in a debate with Ernst Gombrich and Rudolf Arnheim on the nature of pictorial representation, in which the perceptual status of what is pictured in a picture and its recognition was the topic; see Gibson (1971), Gombrich, Gibson, and Arnheim (1971). My acknowledgements go out to Alfred Nordmann for highlighting the subtleties of this debate.
} 
The primary issue here is not the naturalness vs. artificiality of the illusions and misperceptions in question - although, quite obviously, (pi 1 \& 2) are based on artefacts, whereas illusions of kind ( $p i$ 3) occur naturally (while typically being investigated in laboratory settings), and misperceptions of kind ( $p i$ 4) might do so as well. More fundamental than this distinction, and not quite coextensive with it, is the one between perceptual illusions and misperception and their normative status.

In distinguishing illusion from misperception, I am following a fairly straightforward and presumably common-sense distinction discussed by David Armstrong (1960, p. 4) in his interpretation of George Berkeley's theory of vision (1709): If I see something as red, round and having all the visual qualities of a tomato, and it turns out not to be a tomato but a plastic replica, I am likely to have misperceived it, for having mistaken it for another thing on the grounds of some similarity in perceivable surface properties. Still, I have not fallen for a visual illusion - that is, unless I was mistaken about the replica's redness, roundness, etc. to begin with. If, in turn, conditions in the environment or my sense organs are such that I see something as cubic, purple and perhaps lacking other visual qualities of a tomato, and it turns out to be a very normally shaped and coloured fruit of that kind, I have been subject to visual illusion or hallucination respectively, in not getting the surface properties of the object right.

In either case (pi 3 \& 4), I might be at a disadvantage, and it seems natural to assume that this is the standard, the statistically normal result of instances of misperception, hallucination and illusion. If, by Gibson's theory, information for perception is supposed to specify an environment for a perceiving organism, it seems self-suggesting to characterize any divergence between what is perceived and how the environment stands as a failure, and to look either for deficiencies and dysfunctions in the organism's perceptual system or for inconducive or even treacherous conditions in the environment as the causes of that failure. This is what Gibson does in The Senses Considered as Perceptual Systems (1966, Ch. 14), and in his discussion of the difference between natural perception and hallucination (1970).

However, not every divergence between instances of perception and how the world stands needs to amount to unsuccessful perception. The case is clear for hallucination but not for perceptual illusions. Perceptual illusions do not automatically result in misperception, and they may not be maladaptive per se-as Gibson's discussion of pictures as illusions that nonetheless preserve some of the information pertaining to their subject matter should have demonstrated. In naturally occurring perceptual illusions, the normative qualities of my perceptual relation are determined by the actual match or mismatch between the information that is present and the successful realization of my abilities to act upon conditions in my environment, not the illusionary appearances. Getting all the physical properties of an object right might not be all that important in this respect (properties that Gibson did not care much about either). This is the basic idea brought forward by one relatively recent theory in cognitive psychology that has come to be known as the "Empirical Strategy" or, 
less memorably but more descriptively, the "wholly empirical approach to perception" (Purves, Lotto, Williams, \& Xang, 2001; Purves, Wojtach, \& Lotto, 2011). ${ }^{4}$

In combining an environment-directed outlook with a distinctive set of computational and statistical methods, the Empirical Strategy seeks to explain the peculiarities of perception by reference to a history of interactions between organisms and conditions in their environments. The Empirical Strategy is termed "empirical" precisely for rooting the character of perceptions in past experience of the individual or the species. How something is perceived, out of a spectrum of variant possibilities, is determined by how it has been acted upon - and not merely on how it has been perceived - in the past. Past success in doing something in response to an instance of perception will act as a necessary condition in determining how the object or scene in question are being perceived at present.

The authors commence with a discussion of the "inverse optics problem," as it was formulated in George Berkeley's Essay Towards a New Theory of Vision (1709). Berkeley's initial observation was that distances cannot be directly perceived, and that space as such cannot be seen, and that similar conditions apply to other perceptual qualities, such as the magnitude of objects. All that can be directly perceived, on Berkeley's view-and much in contrast to what Gibson would mobilize against this long-dominant view of perception-is the two-dimensional and thus depth-free retinal image. Identical retinal images can be caused by various objects, under various conditions, in various constellations. In view of this problem, Berkeley's proposal was to ground the ability to perceive distances and magnitudes of objects, and in fact any spatial arrangement, in the perceiving subject's experience. Only from experiencing certain objects in certain constellations, one learns to associate prima facie identical visual cues with different perceptual situations, and hence to reliably identify the correct distance, magnitude, etc. of objects.

Purves and his colleagues follow Berkeley's lead when identifying the inverse optics problem as the issue "that light stimuli cannot specify the objects and conditions in the world that caused them" (Purves, Wojtach, \& Lotto, 2011, p. 15588), and, like Berkeley, they delegate the task of specification to the perceiving subject's experience. Their solution to this problem, however, is not as exclusively based on subjective experience as is Berkeley's, and it certainly does not follow Berkeley's idealism. Instead, they adopt a more externalist and supra-individual stance. Conversely, with Gibsonian ecological psychology, the Empirical Strategists share an emphasis on the relation between perception and environmentally embedded behaviour and a markedly realist outlook.

The core of the Empirical Strategists' argument can be parsed into three steps: To begin with, "proximal stimuli trigger patterns of neuronal activity that have been shaped solely by the past consequences of visually guided behaviour" (Purves, Wojtach, Lotto, \& Xang, 2001, p. 285). These consequences in turn are evaluated purely in terms of adaptive success

\footnotetext{
${ }^{4}$ I have to thank Brian McLaughlin for introducing me and others to the Empirical Strategy during the workshop "Perception and Knowledge" at the University of Graz, Austria, in October 2012, and I have to thank Martina Fürst and Guido Melchior, the organisers, for placing that workshop and McLaughlin's paper right when and where I needed it.
} 
rather than any correspondence with the measurable physical properties of the perceived objects. Hence, the measurable physical properties of some object only provide a set of boundary conditions that underdetermine the possible ways of perception. At any rate, they are not represented in vision (Purves, Wojtach, \& Lotto, 2011, p. 15592).

In this circumscribed sense, the Empirical Strategists share Gibson's view that perception is not a process of representing the physical properties of some object or scene but an interaction with some concrete object in its concrete context under concrete conditions of perception. What one can do with or about that object in a given situation or, in Gibsonian terms, what that object affords to the organism is what fixes the way in which it is perceived. Where Gibson focuses on concrete situations of perception and real possibilities, and hence on the present and near future, the Empirical Strategists look backwards in time, seeking to determine how a certain type of perceptual situation came about throughout the natural history of the preceving organisms in their environments.

As conceived under the Empirical Strategy, the relation between perceptual qualities and their target appears at once rather simple and fairly complex and abstract: Some perceptual token will be reproduced if it is closely associated with successful behaviours towards its source or towards some correlate of that source. This condition is sufficient for a very specific sort of empirical adequacy. This empirical adequacy is very specific because it does not build upon any direct relation - and perhaps not even a proper covariance- between a perceptual quality and the physical properties of the perceived object. Divergence between perceptual qualities and the physical conditions at the source does not amount to misperception: "Since the measured properties of objects are not perceived, they cannot be misperceived" (Purves, Wojtach, Lotto, \& Xang, 2001, p. 296).

Instead, what is decisive for getting things right in perception are the frequency distributions of variant retinal patterns, which are mapped not onto variance in physical variables but onto variance in behaviours that differentially respond to certain world affairs, in accordance with the probability distributions of the occurrence of these affairs (Purves, Wojtach, \& Lotto, 2011, p. 15594). For example, the observable mismatch between differences in lightness or brightness of an object as perceptual qualities on the one hand and measured illumination and luminance of the physical objects on the other is attributed to the relation between two factors:

(f 1) Frequency distributions can be determined for variant luminance values of some object as they obtain for the contexts of the various natural scenes in which it, individually or as a member of a type, appears. Objects of some kind will be more often encountered under certain lighting conditions than under others.

(f 2) Frequency distributions are assumed for the rates of success of behaviours of the perceiving organism or his ancestors towards that kind of object under variant conditions. These frequency distributions will be affected by processes of selection, on phylogenetic or ontogenetic levels, of variant behaviours. 
These two types of frequency distributions can be mapped onto each other, so as to see how reliable behavioural success with respect to the object in question can be expected to be under the predominant conditions of appearance in the perceiving organism's environment, and what the cost of failure under less frequent conditions might be. The general strategy is to match perceived qualities of some object against databases of frequencies of occurrence of retinal images corresponding to commonly occurring natural scenes containing that kind of object.

In the present example, equally luminant objects or patterns are perceived as darker when placed in a brightly illuminated context and lighter when placed in a darker context because the differential rates of occurrence of retinal projections caused by the same objects under lighter vs. darker conditions are matched by adaptive behaviours towards those same objects under the respective conditions (Purves, Wojtach, \& Lotto, 2011, p. 15589). At first, the probability distributions of luminance values of objects under lighter vs. darker conditions and the perceived brightness will seem to be 'skewed' towards greater perceived brightness than measurement of luminance would suggest for those retinal patterns which occur most often, namely under poor lighting conditions. However, perception is not skewed in terms of the behaviours that respond to world affairs as they are encountered under these conditions. There will be some use to perceiving objects as exceedingly bright under poor lighting conditions. The use of this seeming illusion should be expected to lie in more reliable or efficient recognition of the kind of object in question when lighting conditions are poor, and hence in more reliable responses to the presence or behaviour of that object. Similar conditions apply to other seeming illusions, such as illusions of length or colour.

Ecological psychologists after Gibson have offered partly similar accounts of the relation between illusion and misperception. With reference to the Ames' distorted room illusion, Sverker Runeson (1988) argues that prima facie illusions are easily corrected for in the course of perceptual activity, and that, in order to persist, illusions typically have to be meticulously created and maintained - which usually does not occur under natural circumstances. Perceptual ambiguity in terms of equivalent configurations in the optic array may be geometrically possible, but these configurations are either physically impossible or never encountered in an unmanipulated environment. Either way, Runeson argues, these equivalent configurations would be informationally irrelevant. Moreover, any residual ambiguity that might get in the way of correctly perceiving affordances is likely to be mended in the further course of perceptual investigation, and in developing one's perceptual skills.

Perceptual learning might allow for correct judgment about some state of affairs even when an illusion persists, argue John Kennedy and colleagues with respect to geometric illusions (Kennedy, Green, Nicholls, \& Liu, 1992): When presented with two well-known size illusions (the Jastrow curves and the Sander parallelogram), transformations of the respective shapes that correspond to perceptual investigation of an object from different angles allowed the majority of the subjects in the experiments to make correct judgements about the true size ratios between the shapes - without the size illusion actually being dispelled. One shape still looked larger than the other but now was known to be of the same 
size. Although Kennedy et al. (1992) do not make that inference, one should expect that an affordance related to the objects in question would have come to be correctly perceived despite the persistence of the visual illusion.

An even more direct match between an ecological view and the argument of the Empirical Strategists is provided by Qin Zhu and Geoffrey Bingham (2011): One of the most robust natural perceptual illusions is the size-weight illusion, in which an object will be perceived as being heavier if and when it is smaller than another object of equal mass. That illusion, the authors seek to demonstrate in an experiment, has a correlate in human subjects' learning of perceiving throwing affordances in terms of selecting objects for optimal sizeweight ratios. These ratios are correctly chosen by proxy of a biased perceived quality. On the background of an evolutionary argument, it is concluded that the illusion has a function in terms of guiding human beings to pick objects that are optimally throw-able over long distances, which was a highly relevant skill in Palaeolithic hunter-gatherer societies, and which has been identified as an ability as uniquely human as language.

In light of these observations on the possible adaptive functions of mismatches between measured physical variables and perceptual qualities, should these mismatches still count as instances of misinformation? Given the relation between the perception of affordances and the notion of natural information that I suggested was held by Gibson, there might be a good reason for believing otherwise: To the extent that a (hypothetical or real) external observer could apply point-to-point mappings, in terms of a multi-dimensional mathematical function, between, first, the variable as it is being registered in any perceptual situation that falls within the range of what the perceiving organism is adapted to, second, the variable as it can be measured by that external observer, third, the concrete conditions under which the ability to perceive that variable in a certain way is realized, and, fourth, the adaptive function of that ability under the given type of conditions, the information that is seemingly misrendered is actually preserved.

Any apparent mismatch between perceived qualities and measurable variables detectable in perception can be resolved, at least in principle, by accounting for the very situation in which perception occurs and the adaptive function of the mapping that applies to this type of perceptual situation. If, under certain conditions, something looks longer, brighter, etc. to the organism than measurement would suggest, these conditions and the functions which that seeming departure serves override correspondence with physical variables. More precisely, adding a "condition" and a "function" variable to the equation will keep the informational mappings intact and allow for an, albeit complex and indirect, correspondence to the values of the physical variables - if and when perception works normally.

Under a genuinely ecological perspective, and in keeping with the strategy of the Empirical Strategy, perceived qualities will be overridden by the informational relations on which successful activities turn out to be grounded. Genuine misperception will only occur if, returning to Warren's above-mentioned case, the subject perceives a stair to be climbable when it is, in fact, not climbable, or vice versa, or if he mistakes an exhaustingly steep 
stairway for a conveniently climbable one. ${ }^{5}$ The subject will then misperceive the respective affordances - which does not even need to imply that the subject is mistaken about the absolute physical measures of the object. Conversely, perceptual illusion will occur if the subject turns out to be mistaken about absolute, extrinsically measured riser heights or transformations of heights in the course of the experiment - which, if the experimental setup duly matches normal conditions for perception, is unlikely to negatively affect perception of the affordance. Hence, perceptual illusions and misperceptions of affordances may coincide but are independent issues. In some cases, perceptual illusions may even contribute to the successful perception of an affordance.

\section{Conclusion}

Although the preceding discussion suggests that the Empirical Strategy in some respects elegantly complements the ecological view, two notable limitations to this approach should be mentioned here: First, almost all examples discussed by the authors are concerned with cases in which identical targets look different to the perceiver under different conditions, whereas their point of departure, the inverse optics problem, was identified by Berkeley as the problem that different targets, when placed in a certain relation to the perceiver, will look identical. Berkeleyan ambiguity does not imply that perception is at variance with the measurable properties but that it becomes ambiguous by one retinal image being in accordance with different instantiations of one specific measurable property. The Empirical Strategy thus accounts for only one of two types of cases of illusion (see Purves, Wojtach, \& Lotto, 2011, p. 15590). Second, the Empirical Strategy remains exclusively concerned with isolated perceptual qualities, such as brightness or perceived angles, length, and motion rather than more complex perceptual affairs in which seeming illusions are mended in the course of perceptual investigation, and in which an object with complex and perhaps multi-modal perceptual qualities is relevant to the organism in certain ways. In contrast, Gibsonian ecological psychology refers to the environmental context and active perception, and the additional information they provide to the organism, so as to compensate for perceptual ambiguities of the second, the Berkeleyan kind.

Despite these limitations, the Empirical Strategy is correct in suggesting that a perceptual illusion, in terms of a prima facie mismatch between perceptual quality and measurable values for some physical variable, may serve adaptive functions. A certain class of perceptual illusions, one that is not clearly distinguished from instances of misperception by Gibson, can thus be accounted for. A necessary precondition for the accomplishment of the adaptive function of a perceptual illusion will be that the illusion is embedded in the context of an environment that is stable enough to allow the organism to handle world

\footnotetext{
${ }^{5}$ In fact, as Warren (1984, p. 695) reports, a stairway can be just as exhaustingly gentle, with low raisers and deep treads, as it can be exhaustingly steep. The perceived gentleness of ascent may indeed be a common misperception of an affordance.
} 
affairs with some reliability and within the bounds of his constitution and abilities. Genuine misperception only occurs if and when either the conditions within organism or environment depart from what he is adapted or accustomed to, or if and when the perceiving organism fails to grasp what can or should be done with the object in question. Perceptual illusions, if and when they have acquired an adaptive function, may actually both be a constituent of the perception of affordances and provide accurate information about some world affair to the perceiving organism. Under normal circumstances, perceptual illusions of the kinds discussed above, for their very regularity and their possible adaptive function, have precious little to do with the misperception of affordances but, very much to the contrary, with helping the organism to get right about some object what he needs to get right, namely what to do with it.

If, in turn, studies in the measurement of affordances are right in assuming that the information relevant to perception normally is intrinsic, the perceiving organism's successful grasp of his relation to the environment will override any apparent mismatch between perceptual qualities and the absolute values for some physical variable that could be measured by an external observer. Misperception, then, can only be misperception of objects in their entirety, not of their measured properties. In Gibson's terms, such misperceptions would be misperceptions of affordances, not, for example, misperceptions of spatial relations or colours. After all, perception, even if it can be assessed in terms of measurement of physical variables by an external observer, does not amount to such measurement by and for the perceiving organism.

This observation will fit rather well with Gibson's contention that we do not perceive such abstract relations as space - a contention that grounds his ecological approach (Gibson, 1979 , p. 32). It is the ecological environment rather than an, abstractly and formally described, physical space that is directly and objectively given to the organism, and that he acts upon. He is perfectly entitled to get things wrong in terms of conditions in physical space as long as he gets them right in terms of the ecology of perception. Still, the ecological environment, despite being objective in nature, cannot be defined in abstraction from the organism inhabiting it. He contributes to the informational relations that he uses. His contributions, if regular in form and adaptive in function, will neither make that information a subjective affair, nor do they give rise to the misperception of affordances.

\section{References}

Armstrong, D. M. (1960). Berkeley's theory of vision: A critical examination of bishop Berkeley's Essay towards a new theory of vision. Melbourne, Autralia: Melbourne University Press.

Berkeley, G. (1709). An essay towards a new theory of vision. Dublin, Ireland: Rhames and Pepyat.

Boumans, M. (2013, November). Affordance affords measurement. Paper presented at "What Affordance Affords" workshop, TU Darmstadt, Germany. 
Chemero, A. (2003a). An outline of a theory of affordances. Ecological Psychology, 15(2), 181195. https://doi.org/10.1207/S15326969ECO1502_5

Chemero, A. (2003b). Information for perception and information processing. Minds and Machines, 13, 577-588. https://doi.org/10.1023/A:1026209002908

Costall, A. (2004). From direct perception to the primacy of action: A closer look at James Gibson's ecological approach to psychology. In G. Bremner \& A. Slater (Eds.), Theories of infant development (pp. 70-89). Oxford, UK: Blackwell. https://doi.org/10.1023/A:1026209002908

de Wit, M. M., van der Kamp, J., \& Withagen, R. (2019). Visual illusions and direct perception: Elaborating on Gibson's insights. New Ideas in Psychology, 36, 1-9. https://doi.org/10.1016/j.newideapsych.2014.07.001

Dretske, F. (1981). Knowledge and the flow of information. Cambridge, MA: MIT Press.

Dretske, F. (1986). Misrepresentation. In R. J. Bogdan (Ed.), Belief. Form, content, and function (pp. 17-36). Oxford, UK: Clarendon Press.

Fodor, J., \& Pylyshyn, Z. (1981). How direct is visual perception? Some reflections on Gibson's 'ecological approach'. Cognition, 9, 139-196. https://doi.org/10.1016/0010-0277(81)90009-3

Gibson, E. J., \& Walk, R. D. (1960). The 'visual cliff'. Scientific American, 202(4), 64-71. https://doi.org/10.1038/scientificamerican0460-64

Gibson, J. J. (1960). The information contained in light. Acta Psychologica, 17, $23-30$. https://doi.org/10.1016/0001-6918(60)90003-2

Gibson, J. J. (1966). The senses considered as perceptual systems. Boston, MA: Houghton Mifflin.

Gibson, J. J. (1970). On the relation between hallucination and perception. Leonardo, 3(4), 425427. https://doi.org/10.2307/1572259

Gibson, J. J. (1971). The information available in pictures. Leonardo, 4, 27-35. https://doi.org/10.2307/1572228

Gibson, J. J. (1973). On the concept of 'formless invariants' in visual perception. Leonardo, 6(1), 43-45. https://doi.org/10.2307/1572424

Gibson, J. J. (1979). The ecological approach to visual perception. (Reprint edition 1986). New York, NY: The Psychology Press.

Gombrich, E., Gibson, J. J., \& Arnheim, R. (1971). Exchange of letters. Leonardo, 4, 195-203. https://doi.org/10.2307/1572214

Heft, H. (2001). Ecological psychology in context: James Gibson, Roger Barker, and the legacy of William James's radical empiricism. Mahwah, NJ: Lawrence Erlbaum Associates. https://doi.org/10.4324/9781410600479

Kennedy, J. M., Green, C. D., Nicholls, A., \& Liu, C. H. (1992). Illusions and knowing what is real. Ecological Psychology, 4(3), 153-172. https://doi.org/10.1207/s15326969eco0403_2

Millikan, R. G. (2001). What has natural information to do with intentional representation? In D. M. Walsh (Ed.), Naturalism, Evolution and Mind (pp. 105-125). Cambridge, UK: Cambridge University Press. https://doi.org/10.1017/CBO9780511563843.006 
Purves, D., Lotto, R. B., Williams, S. M., \& Xang, Z. (2001). Why we see things the way we do: evidence for a wholly empirical strategy of vision. Philosophical Transactions of the Royal Society of London, B, 356, 285-297. https://doi.org/10.1098/rstb.2000.0772

Purves, D., Wojtach, W. T., \& Lotto, R. B. (2011). Understanding vision in wholly empirical terms. Proceedings of the National Academy of Sciences of the United States of America, 108(Supplement 3), 15588-15595. https://doi.org/10.1098/rstb.2000.0772

Reed, E. S. (1988). James J. Gibson and the psychology of perception. New Haven, CT: Yale University Press.

Runeson, S. (1988). The distorted room illusion, equivalent configurations, and the specificity of static optic arrays. Journal of Experimental Psychology: Human Perception and Performance, 14(2), 295-304. https://doi.org/10.1037/0096-1523.14.2.295

Sayre, K. M. (1983). Some untoward consequences of Dretske's "causal theory" of information. Behavioral and Brain Sciences, 6, 78-79. https://doi.org/10.1017/S0140525X00014813

Turvey, M. (1992). Affordances and prospective control: an outline of an ontology. Ecological Psychology, 4(3), 173-187. https://doi.org/10.1207/s15326969eco0403_3

Turvey, M. (2013, November). Affordance: towards an ontology for all organisms. Paper presented at "What Affordance Affords" workshop, TU Darmstadt, Germany.

Turvey, M., \& Shaw, R. (1979). The primacy of perceiving: an ecological reformulation of perception for understanding memory. In L.-G. Nilsson (Ed.), Perspectives on Memory Research: Essays in Honor of Uppsala University's 500th Anniversary (pp. 167-222). Hillsdale, MI: Lawrence Erlbaum Associates.

Turvey, M., Shaw, R. E., Reed, E. S., \& Mace, W. M. (1981). Ecological laws of perceiving and acting: In reply to Fodor and Pylyshyn. Cognition, 9, 237-304. https://doi.org/10.1016/00100277(81)90002-0

van Dijk, L., Withagen, R., \& Bongers, R. M. (2015) Information without content: a Gibsonian reply to enactivists' worries. Cognition, 134, 210-214. https://doi.org/10.1016/j.cognition.2014.10.012

Warren, W. J. J. (1984). Perceiving affordances: visual guidance of stair climbing. Journal of Experimental Psychology, 10(5), 683-703. https://doi.org/10.1037/0096-1523.10.5.683

Withagen, R., \& Chemero, A. (2009). Naturalizing perception: Developing the Gibsonian approach to perception along evolutionary lines. Theory \& Psychology, 19(3), 363-389. https://doi.org/10.1177/0959354309104159

Zhu, Q., \& Bingham, G. P. (2011). Human readiness to throw: The size-weight illusion is not an illusion when picking the best objects to throw. Evolution and Human Behavior, 32, 288-293. https://doi.org/10.1016/j.evolhumbehav.2010.11.005

The editorial and publishing process of this publication has been financed by the Ministry of Science and Higher Education from the funds for the dissemination of research (DUN) within the framework of publishing activity, contract no. 711/P-DUN/2019, period of implementation: the years 2019-2020. 